Check for updates

Cite this: RSC Adv., 2018, 8, 23875

\title{
Complete assignment of the vibrational spectra of borazine: the inorganic benzene
}

\begin{abstract}
Stewart F. Parker (D)
Borazine continues to be relevant in industries as diverse as energy utilisation via fuel cells and as a possible route to boron nitride. Despite it having been known for almost a century, the vibrational spectroscopy of borazine is still incomplete. The inclusion of inelastic neutron scattering spectra has enabled the observation of all of the internal modes of borazine (including the infrared and Raman forbidden modes) for the first time. A complete assignment has been generated with the use of dispersion corrected DFT calculations. This has shown that the accepted ordering of the modes is incorrect in some cases and rationalised conflicting assignments in the literature.
\end{abstract}

Received 6th June 2018

Accepted 26th June 2018

DOI: $10.1039 / c 8 r a 04845 b$

rsc.li/rsc-advances

spectra of the solid state for the first time and to assign these with periodic density functional theory calculations. The comparison of observed and calculated spectra has provided a complete assignment. INS spectroscopy has no selection rules, so all modes are allowed and the infrared and Raman inactive modes are observed for the first time. product of the reaction of diborane and ammonia. It is isostructural and isoelectronic with benzene and, like benzene, is a colourless liquid under ambient conditions. The apparent similarity to benzene has led to borazine being called the 'inorganic benzene', ${ }^{2}$ although this view is not universally accepted. ${ }^{3}$

Borazine is of current interest for two reasons: boron nitride and hydrogen storage. It can be used as a carbon-free precursor to boron nitride, with the advantage that the initial condensation to polyborazylene results in a processable material. ${ }^{4}$ Borazine is also used in preparative routes using chemical vapour deposition. ${ }^{5}$ In the area of hydrogen storage materials, borazine is an unwelcome by-product ${ }^{6}$ of the dehydrogenation of ammonia borane because it rapidly poisons the low temperature fuel cells that are often the destination of the released hydrogen.

The vibrational spectroscopy of borazine has been investigated many times over the years. However, the work has almost exclusively been concerned with gas phase infrared studies. ${ }^{7-11}$ The molecule offers multiple possibilities for isotopic substitution: ${ }^{1,2} \mathrm{H},{ }^{10,11} \mathrm{~B}$ and ${ }^{14,15} \mathrm{~N}$ and many of the possible combinations have been examined. Apart from a brief conference report of the solid state infrared spectrum, ${ }^{12}$ there are no reported solid state spectra. The molecule has been analysed by normal coordinate analysis using empirical force fields ${ }^{13,14}$ and also by an $a b$ initio study. ${ }^{15}$ The analysis of the spectra is greatly complicated by the fact that the free molecule has $D_{3 \mathrm{~h}}$ symmetry (as shown by gas phase electron diffraction ${ }^{16}$ ) which results in three silent modes.

The purpose of the present work was to measure the vibrational infrared, Raman and inelastic neutron scattering (INS) ${ }^{17}$

\section{Experimental}

Borazine was obtained from Gelest and used as received. All operations involving transfer of borazine were conducted in a glovebox to avoid hydrolysis. Infrared spectra $\left(2 \mathrm{~cm}^{-1}\right.$ resolution, $256 \mathrm{scans}$ ) were recorded between $105 \mathrm{~K}$ and $298 \mathrm{~K}$ with a Bruker Vertex 70 Fourier transform infrared spectrometer using a Specac single reflection variable temperature attenuated total internal reflection accessory. Raman spectra were recorded in the range $13-235 \mathrm{~K}$ with a previously described ${ }^{18}$ Renishaw InVia system using $785 \mathrm{~nm}$ excitation and have been corrected for the instrument response. In this configuration the largest Raman shift obtainable is $\sim 3200 \mathrm{~cm}^{-1}$. INS spectra were recorded at $<10 \mathrm{~K}$ using the TOSCA ${ }^{19}$ and MAPS $^{20}$ spectrometers

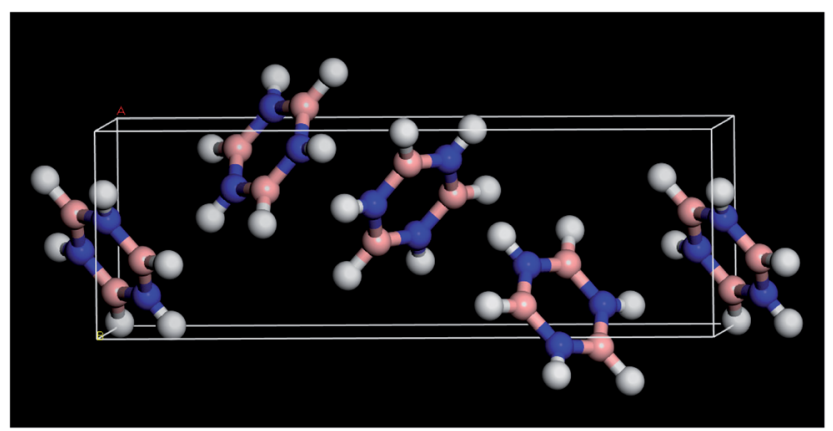

Fig. 1 Solid state structure of borazine. ${ }^{3}$ Blue $=$ nitrogen, pink $=$ boron, white $=$ hydrogen 
at ISIS. $^{21}$ For TOSCA, only the data from the backscattering detectors was used, because the neutron absorption from ${ }^{10} \mathrm{~B}$ in the natural abundance borazine resulted in no usable data in the forward scattering detectors.

Dispersion corrected periodic density functional theory (DFT-D) calculations were carried out using the plane wave pseudopotential method as implemented in the CASTEP code. ${ }^{2,23}$ Exchange and correlation were approximated using the $\mathrm{PBE}^{24}$ functional with the Tkatchenko-Scheffler (TS) dispersion correction scheme ${ }^{25}$ within the generalized gradient approximation (GGA). The plane-wave cut-off energy was $1000 \mathrm{eV}$. Brillouin zone sampling of electronic states was performed on $10 \times 10 \times 4$ Monkhorst-Pack grid (30 k-points). The equilibrium structure, an essential prerequisite for lattice dynamics calculations was obtained by BFGS geometry optimization after which the residual forces were converged to $\pm 0.00755 \mathrm{eV}^{-1}$. Phonon frequencies were obtained by diagonalization of dynamical matrices computed using densityfunctional perturbation theory ${ }^{26}$ and also to compute the dielectric response and the Born effective charges, and from these the mode oscillator strength tensor and infrared absorptivity were calculated. In addition to the calculation of transition energies and intensities at zero wavevector, phonon dispersion was also calculated along high symmetry directions throughout the Brillouin zone. For this purpose, dynamical matrices were computed on a regular grid of wavevectors throughout the Brillouin zone and Fourier interpolation was used to extend the computed grid to the desired fine set of points along the high-symmetry paths. ${ }^{27}$ Transition energies (assuming the harmonic approximation) for isotopic species were calculated from the dynamical matrix that is stored in the CASTEP checkpoint file using the PHONONS utility. ${ }^{28}$ The atomic displacements in each mode that are part of the CASTEP output, enable visualization of the modes to aid assignments and are also all that is required to generate the INS spectrum using the program ACLIMAX. ${ }^{29}$ It is emphasised that for all the calculated spectra shown the transition energies have not been scaled.

\section{Results}

The vibrational spectra; infrared, Raman and INS, of borazine in the solid state are shown for the first time in Fig. 2. The complementarity of the three techniques is immediately apparent: modes that are strong in one technique are often weak or absent in the others. The transition energies are listed in Table 1. The mode numbering and descriptions are that of Niedenzu et al. ${ }^{10}$

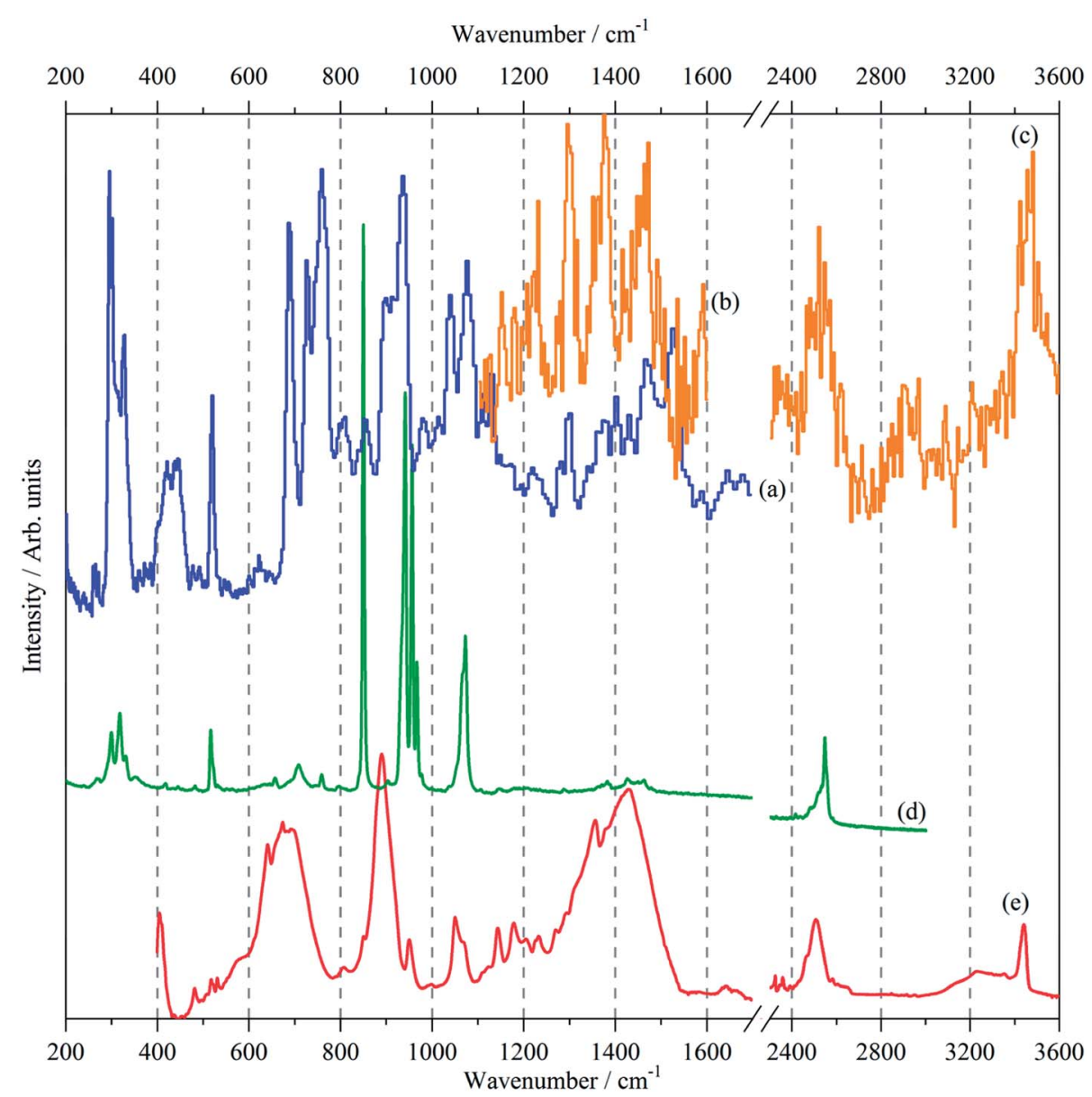

Fig. 2 Vibrational spectra of borazine in the solid state. INS spectra recorded at $10 \mathrm{~K}$ with: (a) TOSCA (b) MAPS, $\left(E_{\mathrm{i}}=3200 \mathrm{~cm}^{-1}\right),(\mathrm{c}) \mathrm{MAPS}\left(E_{\mathrm{i}}=\right.$ $4800 \mathrm{~cm}^{-1}$ ), (d) Raman recorded at $11 \mathrm{~K}$ and (e) infrared recorded at $105 \mathrm{~K}$. 
Table 1 Observed and calculated modes of borazine in the solid state

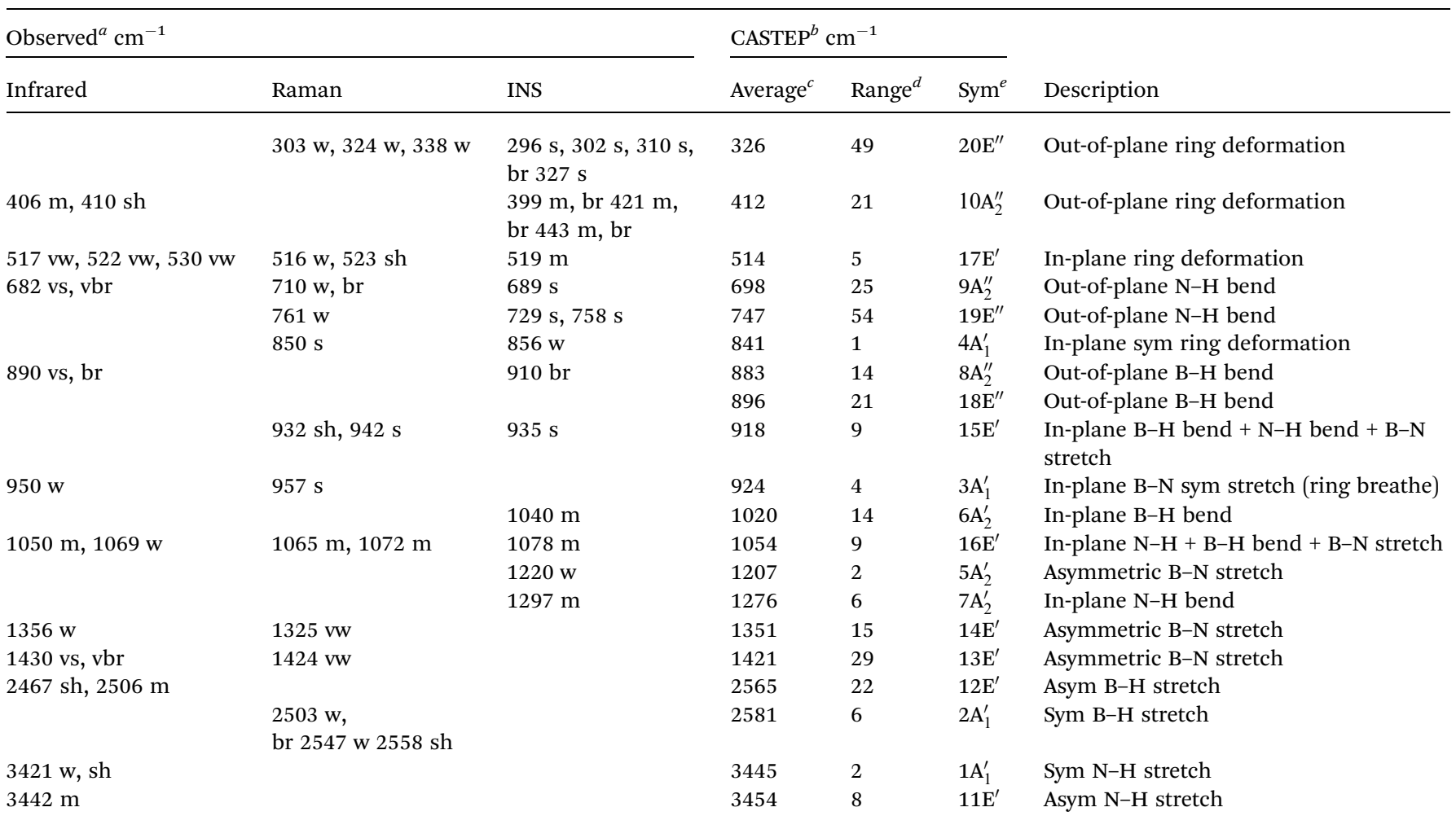

${ }^{a} \mathrm{~s}=$ strong, $\mathrm{m}=$ medium, $\mathrm{w}=$ weak, br $=$ broad, $\mathrm{sh}=$ shoulder, $\mathrm{v}=$ very. ${ }^{b}$ Transition energies at the at the $\Gamma$-point of the complete unit cell containing four molecules. ${ }^{c}$ Average of the factor group split transition energies at the $\Gamma$-point. ${ }^{d}$ Difference between the highest and lowest transition energy of the factor group at the $T$-point. ${ }^{e}$ Mode number and symmetry label for the mode in $D_{3 \mathrm{~h}}$ symmetry.

Variable temperature infrared spectra, Fig. 3, show that the molecule is unchanged between 258 and $105 \mathrm{~K}$, in agreement with the X-ray structure determination. ${ }^{3}$ Further the spectra are almost indistinguishable from that of the liquid state, Fig. 3a. The low temperature, $15 \mathrm{~K}$, Raman spectrum allows a number of very weak bands to be observed, Fig. 4 . The symmetric $\mathrm{N}-\mathrm{H}$ stretch is most easily seen in the Raman spectrum. Unfortunately, with $785 \mathrm{~nm}$ excitation the CCD cuts-off at $\sim 3200 \mathrm{~cm}^{-1}$, consequently it was not observed. However, the infrared spectrum shows a shoulder at $3421 \mathrm{~cm}^{-1}$ on the main asymmetric stretch band. The DFT calculations (see later) show that the symmetric mode is activated in the solid state and the band at $3421 \mathrm{~cm}^{-1}$ is assigned to this mode.

The INS spectra, Fig. $2 \mathrm{a}-\mathrm{c}$ and 5 , show that the solid state is more complicated than the infrared or Raman spectra suggest. This is evidenced by the complex band shapes of the modes in the $300-500$ and $700-800 \mathrm{~cm}^{-1}$ regions. INS spectra have no selection rules, hence all modes are allowed, however, there is a strong "propensity rule" that modes that involve proton motion will dominate the spectra. ${ }^{17}$ Further, INS spectra depend on both energy transfer, $\omega\left(\mathrm{cm}^{-1}\right)$, and momentum transfer, $Q$ $\left(\AA^{-1}\right)$, thus are able to access the entire Brillouin zone, not just the $\Gamma$-point i.e. $Q \sim 0$, as for infrared or Raman spectroscopies. Thus the complex band shapes indicate that there is clearly significant factor group splitting and/or vibrational dispersion present in this system.
In order to better understand this system, periodic density functional theory (DFT) of the complete unit cell was employed. Borazine crystallises in the tetragonal space group, $P 4_{3} 2_{1} 2$, with

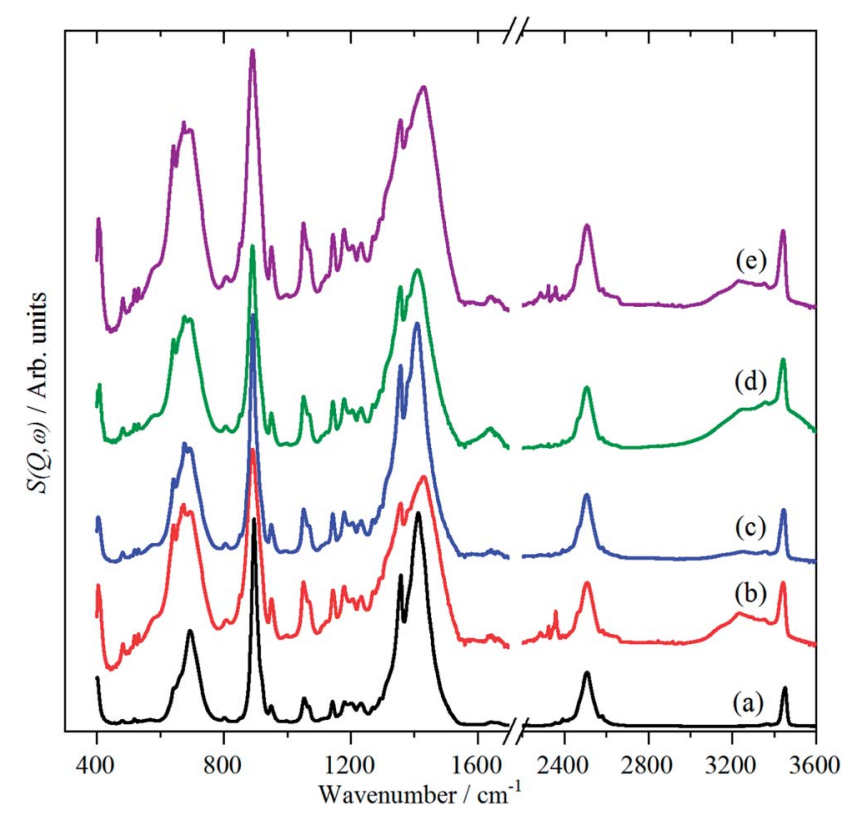

Fig. 3 Vibrational temperature infrared spectra of borazine. (a) liquid at $298 \mathrm{~K}$, solid at: (b) $258 \mathrm{~K}$, (c) $213 \mathrm{~K}$, (d) $160 \mathrm{~K}$ and (e) $105 \mathrm{~K}$. The broad features at 1650 and $3300 \mathrm{~cm}^{-1}$ are due to ice. 


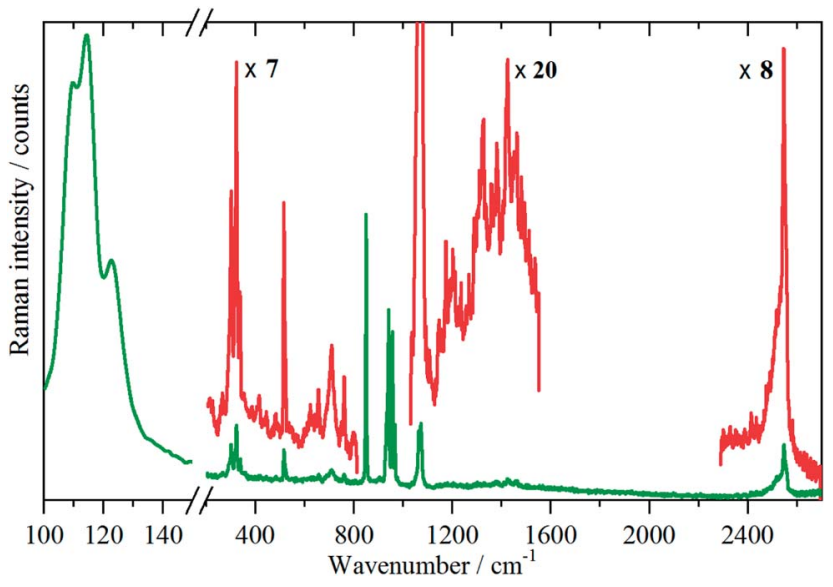

Fig. 4 Raman spectrum of borazine in the solid state at $11 \mathrm{~K}$.

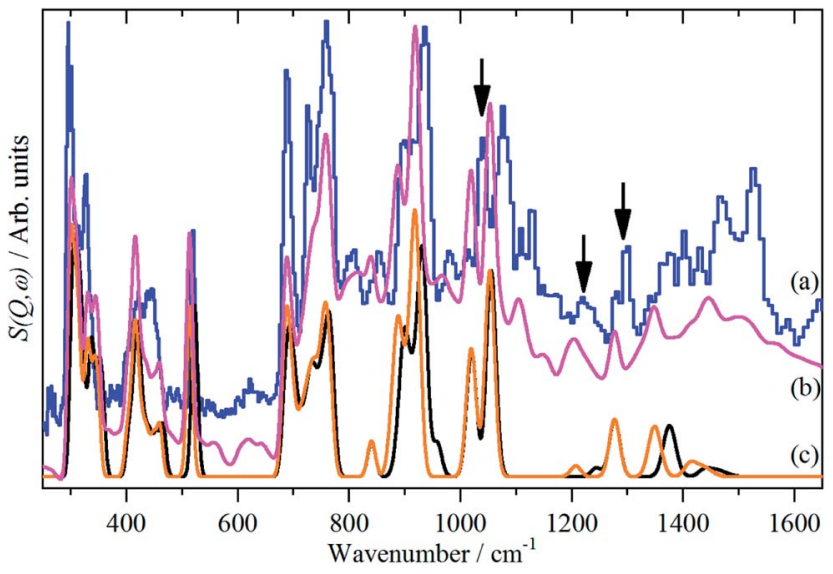

Fig. 5 Comparison of the INS spectra of borazine in the solid state. (a) Experimental (TOSCA) and generated from the CASTEP calculation (b) complete unit cell, $Z=4$, all ${ }^{11} \mathrm{~B}$, all transitions and (c) fundamentals only for all ${ }^{10} \mathrm{~B}$ (black, rightmost trace) and ${ }^{11} \mathrm{~B}$ (orange, leftmost trace). The three infrared and Raman inactive $A_{2}^{\prime}$ modes are indicated by arrows.

four molecules in the unit cell $(Z=4)^{3}$ and this was used as the initial structure for the computational study. A series of calculations, using a range of cut-off energies and $k$-point grids, always resulted in imaginary modes, indicating that the structure was dynamically unstable. The vibrational spectroscopy shows no evidence for a structural phase change, suggesting that the model was inadequate in some way. The problem was resolved by the inclusion of the Tkatchenko and Scheffler (TS) ${ }^{25}$ semi-empirical dispersion correction for DFT. This attempts to account for non-covalent forces, such as hydrogen bonding and van der Waals interactions in the solid state. With the inclusion of the TS correction, all real modes were obtained across the entire Brillouin zone, Fig. 6.

It can be seen that for the modes in the 300-500 and 700$800 \mathrm{~cm}^{-1}$ regions that there is both strong dispersion: the mode at $\sim 400 \mathrm{~cm}^{-1}$ at the $\Gamma$-point has a transition energy of $\sim 450 \mathrm{~cm}^{-1}$ at the $A$ and $M$ points in the Brillouin zone. There is also a large factor group splitting: columns 4 and 5 of Table 1 show the average and the range (defined as highest-lowest transition energy) of the factor group components.

Fig. $5 \mathrm{a}$ and $\mathrm{b}$ compares the observed and calculated INS spectra of borazine in the internal mode region. It can be seen that there is generally good agreement for both the transition energies and their intensities.

\section{Discussion}

Unlike ammonia-borane, $\mathrm{H}_{3} \mathrm{~N}-\mathrm{BH}_{3},{ }^{30}$ the structure shows no evidence for dihydrogen bonding, which is characterised by approximately linear $\mathrm{N}-\mathrm{H} \cdots \mathrm{H}$ bonds and bent $\mathrm{B}-\mathrm{H} \cdots \mathrm{H}$ bonds. ${ }^{30}$ In the geometry optimised structure the shortest $\mathrm{H} \cdots \mathrm{H}$ distance is $2.512 \AA$, outside the accepted range of 1.7-2.2 $\AA$ and larger than twice the van der Waals radius of hydrogen, $2.4 \AA$. Although borazine has a gas phase dipole moment of $<0.1 \mathrm{D}^{16}$ (and zero for the ideal $D_{3 \mathrm{~h}}$ structure), the calculations do show significant charge on the atoms: $\mathrm{N}:-0.92 /-0.21$, B: $+0.52 /+0.15$, $\mathrm{H}(\mathrm{N}):+0.42 /+0.12$ and $\mathrm{H}(\mathrm{B}):-0.01 /-0.06$ electrons for the Mulliken and Hirschfeld charges respectively.

The good agreement between the observed and calculated spectra in Fig. 5, enables unambiguous assignments from the mode visualisations to be made. These are listed in Table 2 and compared to those in the literature. In $D_{3 \mathrm{~h}}$ symmetry, $\mathrm{A}_{1}^{\prime}, \mathrm{E}^{\prime}$ and $\mathrm{E}^{\prime \prime}$ are Raman active, $\mathrm{A}_{2}^{\prime \prime}$ and $\mathrm{E}^{\prime}$ are infrared active and $\mathrm{A}_{2}^{\prime}$ are inactive in both, note that all modes are allowed in the INS spectrum. For the $\mathrm{A}_{1}^{\prime}$ and $\mathrm{A}_{2}^{\prime \prime}$ modes there is general agreement on the assignments and this work confirms these. For the $\mathrm{E}^{\prime}$ modes, there is confusion over the ordering of modes 15 and 16. These are described as in-plane bending modes of B-H and N-H respectively. ${ }^{10}$ The mode animations show that these are in fact more complex and are coupled vibrations involving B-H and $\mathrm{N}-\mathrm{H}$ bending as well as $\mathrm{B}-\mathrm{N}$ stretching. On the basis of the relative amplitudes of the motion, mode 16 is assigned at $1078 \mathrm{~cm}^{-1}$ (N-H bend) and mode 15 at $935 \mathrm{~cm}^{-1}$ (B-H bend).

The assignment of the highest energy $\mathrm{E}^{\prime \prime}$ mode is also debated. It is usually assigned to the weak band at $967 \mathrm{~cm}^{-1}$. However, our calculations show no mode at this energy and the INS spectrum only shows a very weak mode. This is inconsistent with it being the B-H out-of-plane bend, which would have a large amplitude of motion of the proton and hence strong INS intensity. The present work locates this mode at $890 \mathrm{~cm}^{-1}$ (896 $\mathrm{cm}^{-1}$ calculated).

The modes that were least certain are the in-plane $\mathrm{A}_{2}^{\prime}$ modes, 5, 6 and 7, which are described as asymmetric B-N stretching, B-H and $\mathrm{N}-\mathrm{H}$ bending respectively. Fig. 7 shows the atomic displacements for the calculated modes at 1279, 1215 and $1026 \mathrm{~cm}^{-1}$ respectively. It is apparent that these correspond to modes 7,5 and 6.

The INS spectra, Fig. 2a-c and 5, provide the first experimental observation of the modes at 1220, 1040 and $1297 \mathrm{~cm}^{-1}$ for modes 5, 6 and 7 respectively. The only previous claim ${ }^{11}$ was by infrared spectroscopy of matrix isolated borazine. Two weak modes were seen which were believed to be combinations involving modes 6 and 7. This work shows that those assignments are incorrect. 

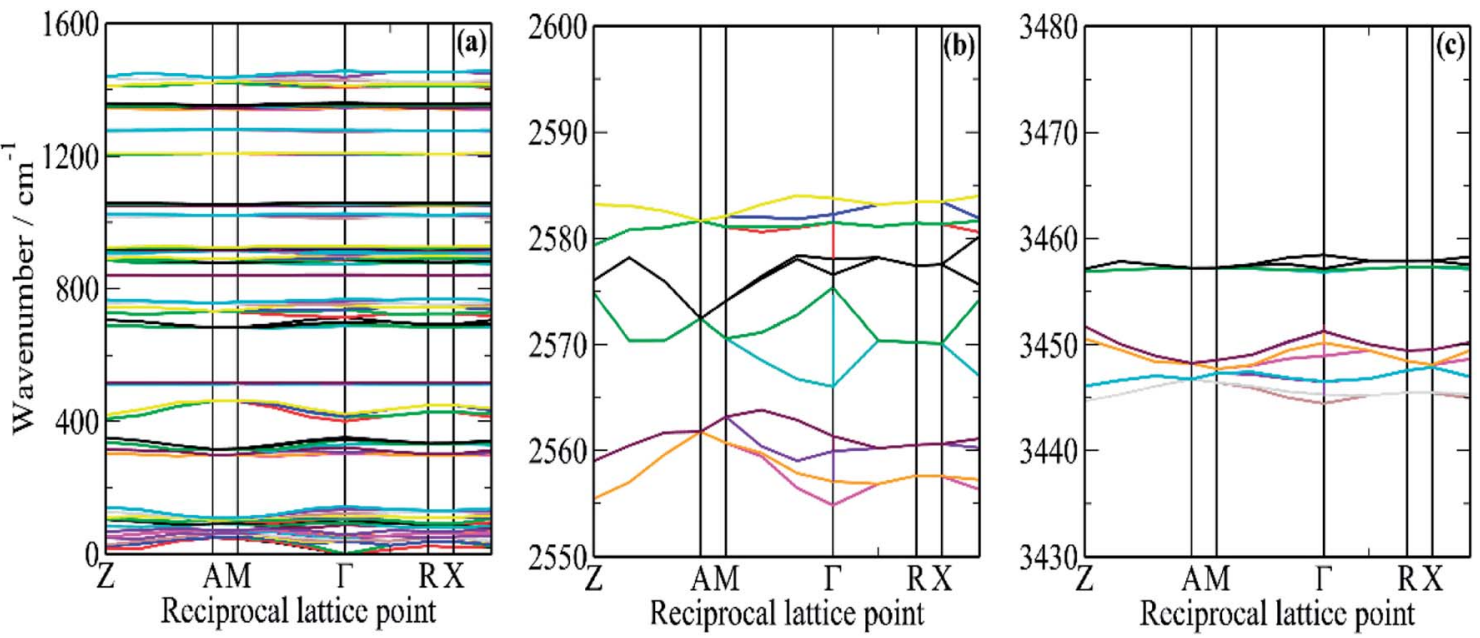

Fig. 6 Dispersion curves of borazine in the solid state generated from the CASTEP calculation. (a) In the external mode and fingerprint region, (b) in the $\mathrm{B}-\mathrm{H}$ stretch region and (c) in the $\mathrm{N}-\mathrm{H}$ stretch region.

Table 2 Comparison of vibrational assignments of borazine in $D_{3 h}$ symmetry

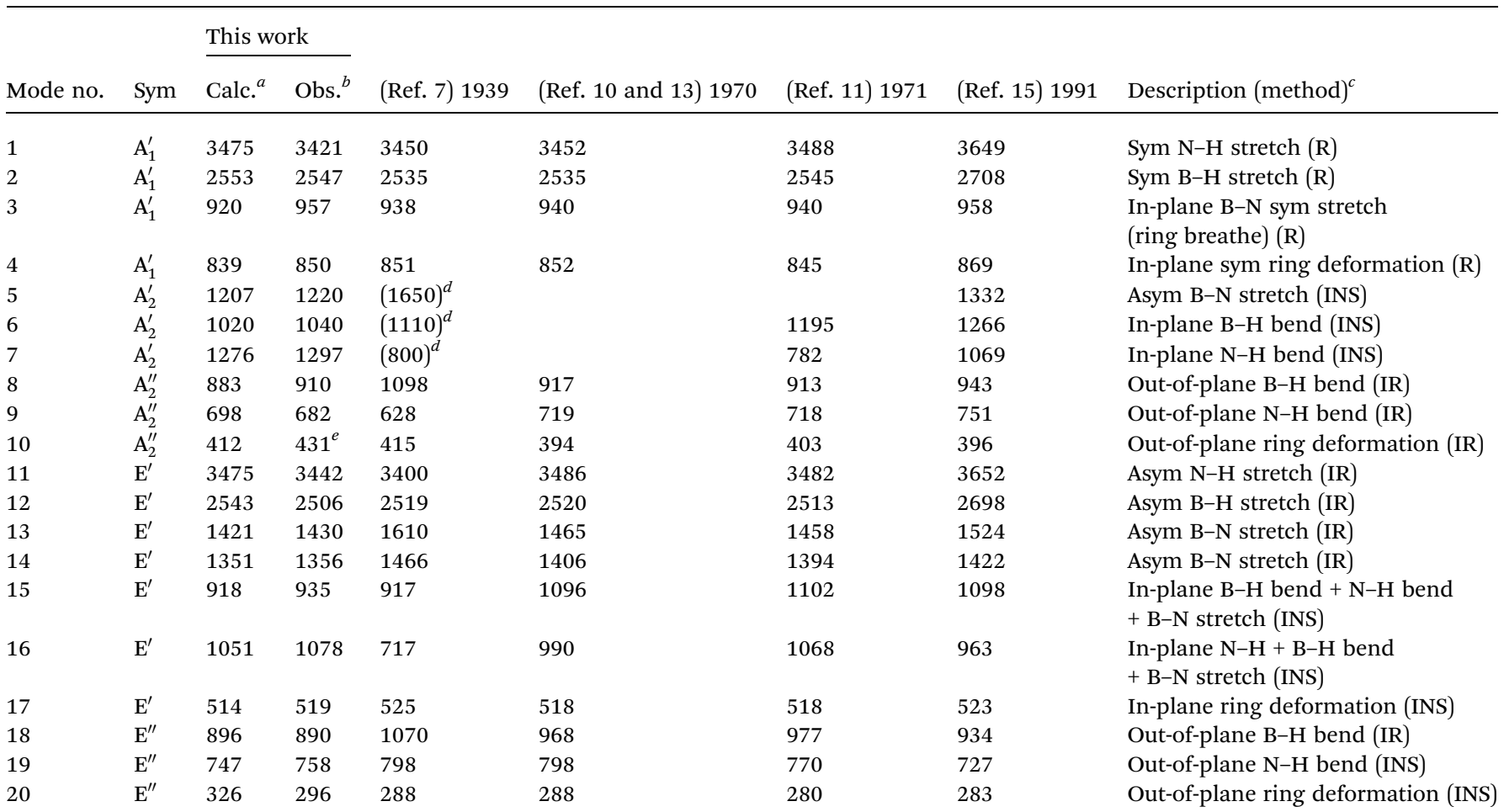

${ }^{a}$ Average of the factor group split transition energies at the $\Gamma$-point. ${ }^{b}$ Transition energy of the strongest mode in the spectra closest in energy to the calculated value. ${ }^{c}$ Calculated from the force field, not observed. ${ }^{d}$ Method is the technique where the mode is best observed; INS (INS), Raman (R) or infrared (IR). ${ }^{e}$ This entry is the centre frequency of the complex line shape resulting from both dispersion and a large factor group splitting in this mode.

Borazine containing natural abundance boron $\left(20 \%{ }^{10} \mathrm{~B}\right.$, $80 \%{ }^{11} \mathrm{~B}$ ) was used for this work. This leads to the question as to whether this is manifested in the spectra. In the gas phase and in a matrix, weak ${ }^{10} \mathrm{~B}$ satellite peaks are observed for some of the modes, ${ }^{\mathbf{9} 11}$ I have shown previously how the isotopic distribution in a crystal may be calculated, ${ }^{31}$ and for natural abundance borazine with four molecules in the primitive cell, the largest constituents are: $\left({ }^{10} \mathrm{~B}^{11} \mathrm{~B}^{11} \mathrm{~B},{ }^{10} \mathrm{~B}^{11} \mathrm{~B}^{11} \mathrm{~B},{ }^{11} \mathrm{~B}^{11} \mathrm{~B}^{11} \mathrm{~B},{ }^{11} \mathrm{~B}^{11} \mathrm{~B}^{11} \mathrm{~B}\right)=$ $23.19 \%$ and $\left({ }^{10} \mathrm{~B}^{11} \mathrm{~B}^{11} \mathrm{~B},{ }^{11} \mathrm{~B}^{11} \mathrm{~B}^{11} \mathrm{~B},{ }^{11} \mathrm{~B}^{11} \mathrm{~B}^{11} \mathrm{~B},{ }^{11} \mathrm{~B}^{11} \mathrm{~B}^{11} \mathrm{~B}\right)=$ $20.62 \%$, the all- ${ }^{11} \mathrm{~B}$ species is only a minor constituent, $6.87 \%$. Fig. 5c shows the calculated INS spectra for the most extreme compositions: all ${ }^{10} \mathrm{~B}$ and all ${ }^{11} \mathrm{~B}$. It can be seen that the difference is small for most of the modes. 


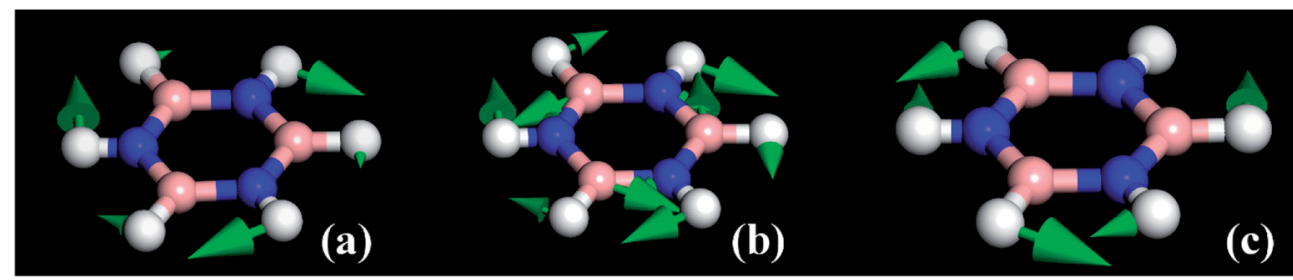

Fig. 7 The atomic displacements for the modes calculated at (a) 1279 , (b) 1215 and (c) $1026 \mathrm{~cm}^{-1}$

\section{Conclusions}

Despite it having been known for almost a century, the vibrational spectroscopy of borazine was still incomplete. The inclusion of INS spectra has enabled the observation of all of the internal modes of borazine for the first time. A complete assignment has been generated with the use of DFT-D calculations. This has shown that the accepted ordering of the modes is incorrect in some cases and rationalised conflicting assignments in the literature.

\section{Conflicts of interest}

There are no conflicts of interest to declare.

\section{Acknowledgements}

The STFC Rutherford Appleton Laboratory is thanked for funding and access to neutron beam facilities. Computing resources (time on the SCARF compute cluster for the CASTEP calculations) were provided by STFC's e-Science facility.

\section{References}

1 A. Stock and E. Pohland, Ber. Dtsch. Chem. Ges., 1926, 59, 2215-2223.

2 N. N. Greenwood and A. Earnshaw, Chemistry of the Elements, Pergamon Press, Oxford, 1984, p. 238.

3 R. Boese, A. H. Maulitz and P. Stellberg, Chem. Ber., 1994, 127, 1887-1889.

4 P. J. Fazen, E. E. Remsen, J. S. Beck, P. J. Carroll, A. R. McGhie and L. G. Sneddon, Chem. Mater., 1995, 7, 1942-1956.

5 K. K. Kim, A. Hsu, X. Jia, S. M. Kim, Y. Shi, M. Dresselhaus, T. Palacios and J. Kong, ACS Nano, 2012, 6, 8583-8590.

6 A. Al-Kukhun, H. T. Hwang and A. Varma, Ind. Eng. Chem. Res., 2011, 50, 8824-8835.

7 B. L. Crawford and J. T. Edsall, J. Chem. Phys., 1939, 7, 223232.

8 E. Silberman, Spectrochim. Acta, 1967, 28A, 2021-2028.

9 V. B. Kartha, S. L. N. G. Krisnamachari and C. R. Subramaniam, J. Mol. Spectrosc., 1967, 23, 149-157.

10 K. Niedenzu, W. Sawodny, H. Watanabe, J. W. Dawson,

T. Totani and W. Weber, Inorg. Chem., 1967, 6, 1453-1461.

11 A. Kaldor and R. F. Porter, Inorg. Chem., 1971, 10, 775-785.
12 E. Huler, E. Silberman and E. A. Jones, Bull. Am. Phys. Soc., 1970, 15, 177.

13 K. Blick, J. W. Dawson and K. Nidenzu, Inorg. Chem., 1970, 9, 1416-1421.

14 E. Huler, E. Silberman and E. A. Jones, Spectrochim. Acta, 1970, 26A, 2241-2246.

15 F. Ramondo, G. Portalone and L. Bencivenni, J. Mol. Struct.: THEOCHEM, 1991, 236, 29-39.

16 W. Harshbarger, G. H. Lee II, R. F. Porter and S. H. Bauer, Inorg. Chem., 1969, 8, 1683-1689.

17 P. C. H. Mitchell, S. F. Parker, A. J. Ramirez-Cuesta and J. Tomkinson, Vibrational spectroscopy with neutrons, with applications in chemistry, biology, materials science and catalysis, World Scientific, Singapore, 2005.

18 M. A. Adams, S. F. Parker, F. Fernandez-Alonso, D. J. Cutler, C. Hodges and A. King, Appl. Spectrosc., 2009, 63, 727-732.

19 D. Colognesi, M. Celli, F. Cilloco, R. J. Newport, S. F. Parker, V. Rossi-Albertini, F. Sacchetti, J. Tomkinson and M. Zoppi, Appl. Phys. A, 2002, 74(suppl.), S64-S66.

20 S. F. Parker, D. Lennon and P. W. Albers, Appl. Spectrosc., 2011, 65, 1325-1341.

$21 \mathrm{http}: / /$ www.isis.stfc.ac.uk.

22 S. J. Clark, M. D. Segall, C. J. Pickard, P. J. Hasnip, M. J. Probert, K. Refson and M. C. Payne, Z. Kristallogr., 2005, 220, 567-570.

23 K. Refson, P. R. Tulip and S. J. Clark, Phys. Rev. B: Condens. Matter Mater. Phys., 2006, 73, 155114.

24 J. Perdew, K. Burke and M. Ernzerhof, Phys. Rev. Lett., 1996, $77,3865$.

25 A. Tkatchenko and M. Scheffler, Phys. Rev. Lett., 2009, 102, 073005.

26 V. Milman, A. Perlov, K. Refson, S. J. Clark, J. Gavartin and B. Winkler, J. Phys.: Condens. Matter, 2009, 21, 485404.

27 X. Gonze, J.-C. Charlier and M. P. Teter, Phys. Rev. B, 1994, 50, 13035-13038.

28 K. Refson, Phonons and Related Calculations in CASTEP, http://www.castep.org/.

29 A. J. Ramirez-Cuesta, aCLIMAX 4.0.1, Comput. Phys. Commun., 2004, 157, 226-238.

30 W. T. Klooster, T. F. Koetzle, P. E. M. Siegbahn, T. B. Richardson and R. H. Crabtree, J. Am. Chem. Soc., 1999, 121, 6337-6343.

31 S. F. Parker, Spectrochim. Acta, Part A, 2015, 140, 462-464. 